\title{
Channel Estimation and Joint Beamforming Design for Multi-IRS MIMO systems
}

\author{
Kenneth Benicio, Bruno Sokal, and André L. F. de Almeida
}

\begin{abstract}
In this paper, we propose a channel estimation and joint beamforming design method for multi-IRS multiple-input and multiple-output (MIMO) systems. We investigate the scenario where multiple intelligent reflecting surfaces (IRSs) are located on the exact spot of the channel scatterers. We investigate the spectral efficiency (SE), benefits and drawbacks of deploying multiple IRSs in comparison with a scenario with a single or no IRS. Our simulation results show a trade-off between the number of reflecting elements and the total number of IRSs in the system.
\end{abstract}

Keywords-Intelligent reflecting surface, MIMO systems, channel estimation, beamforming design.

\section{INTRODUCTION}

Intelligent reflecting surfaces (IRSs) have caught the attention of the wireless communications community in recent years [1]- [2] for being a promising candidate for beyond fifth generation (B5G) and the sixth generation (6G) wireless networks. Usually, an IRS is a 2D-panel [3] composed of $N$ (semi-) passive reflecting elements that apply phase-shifts to the the impinging electromagnetic waves so that they add constructively at the intended receiver, by providing significant signal-to-noise ratio (SNR) gains. The only active element in the IRS structure is the smart controller, which has a negligible power consumption compared to other current technologies such as relays [4]. Also, depending on the phase-shift design of each IRS element, it has been shown that the IRS can provide an SNR gain of order $N^{2}$. The limits of this gain are discussed in [5], where the authors analyze how large an IRS must be, i.e., how many reflecting elements are required to surpass the known relaying schemes.

Although an IRS can offer a very high SNR gain at low energy consumption levels compared to other technologies, one of the main problems is that of channel state information (CSI) acquisition. Since the IRS is fully passive structure with no digital signal processing capability, channel estimation must be performed at the receiver using pilots sent by the transmitter and reflected by the IRS according to predefined patterns. Recent works in the literature have proposed solutions that address this problem such as [6]-[9].

Kenneth Benicio, Bruno Sokal, and André L. F. de Almeida are with the Wireless Telecommunications Research Group (GTEL), Department of Teleinformatics Engineering (DETI), Federal University of Ceará (UFC), Fortaleza, Brazil. E-mails: kennethbrenner4242@gmail.com, \{brunosokal,andre\}@gtel.ufc.br. This work was supported by the Ericsson Research, Sweden, and Ericsson Innovation Center, Brazil, under UFC.48 Technical Cooperation Contract Ericsson/UFC. This study was financed in part by the Coordenação de Aperfeiçoamento de Pessoal de Nível Superior - Brasil (CAPES)-Finance Code 001, and CAPES/PRINT Proc. 88887.311965/201800. André L. F. de Almeida like to acknowledge CNPq for its financial support under the grant 306616/2016-5.
Since the IRS is a recent concept, [10] and [11] tried to explore the physical laws behind the IRS while dispelling some myths around it. In [12] IRSs are used to diminish the impact of coverage holes in mmWave MIMO systems. It is shown that by creating an effective virtual line-of-sight path between the transmitter and the receiver, the IRS can be used to minimize the effects of severe path loss and the high directivity intrinsic to mmWave communications.

Closely related to the this paper are the works [6] and [7] for channel estimation and [13] [14] for joint beamforming design. The authors in [6] propose a tensor modeling approach to obtain the CSI using a supervised method, where two algorithms were formulated to decouple the estimation of the involved channels. The work [7] also presents a tensorbased approach for channel estimation, where the authors propose three different algorithms. Therein, the authors also discuss the beamforming problem. In [13], an optimal channel estimation scheme guided by the minimum variance unbiased estimator is proposed. In [14], the authors discuss different channel estimation algorithms as well as joint IRS phaseshift optimization and active beamforming designs for MIMO systems assisted by an IRS.

In this paper, we consider a MIMO communication system assisted by multiple IRSs. Assuming a specular multipath propagation scenario, we place multiple intelligent reflecting surfaces (IRSs) on the exact spot of the dominant channel scatterers. A channel estimation protocol and a joint passive (IRSs phase-shifts) and active (transmit/receive) beamformings is then proposed for this scenario. We investigate the possible benefits and drawbacks of the proposed multi-IRS system model, in comparison with a scenario with a single or no IRS. In particular, assuming a fixed total number of reflecting elements in the system, we compare a centralized deployment with a single IRS with a distributed one having multiple IRS. Our numerical results show the remarkable spectral efficiency gains of a multi-IRS MIMO communication system.

\section{A. Notation}

Scalars and column vectors are represented by lowercase letters $(a, b, \ldots)$ and boldface lowercase letters $(\mathbf{a}, \mathbf{b}, \ldots)$, respectively. Matrices are symbolized by boldface capital letters $(\mathbf{A}, \mathbf{B}, \ldots) . \mathbf{X}^{*}, \mathbf{A}^{\mathrm{T}}, \mathbf{A}^{\mathrm{H}}$, and $\mathbf{A}^{+}$stand for the conjugate, transpose, Hermitian, and Moore-Penrose pseudoinverse respectively. The $j$ th column of $\mathbf{A} \in \mathbb{C}^{I \times J}$ is denoted by $\mathbf{a}_{j} \in \mathbb{C}^{I \times 1}$. The operator $\operatorname{vec}(\cdot)$ transforms a matrix into a vector by stacking its columns, e.g., $\operatorname{vec}(\mathbf{A})=\mathbf{a} \in \mathbb{C}^{I J \times 1}$, while the unvec $(\cdot)$ operator does in the inverse operation, i.e., unvec $_{I \times J}(\mathbf{a})=\mathbf{A} \in \mathbb{C}^{I \times J}$. The operator $\operatorname{diag}(\cdot)$ converts 


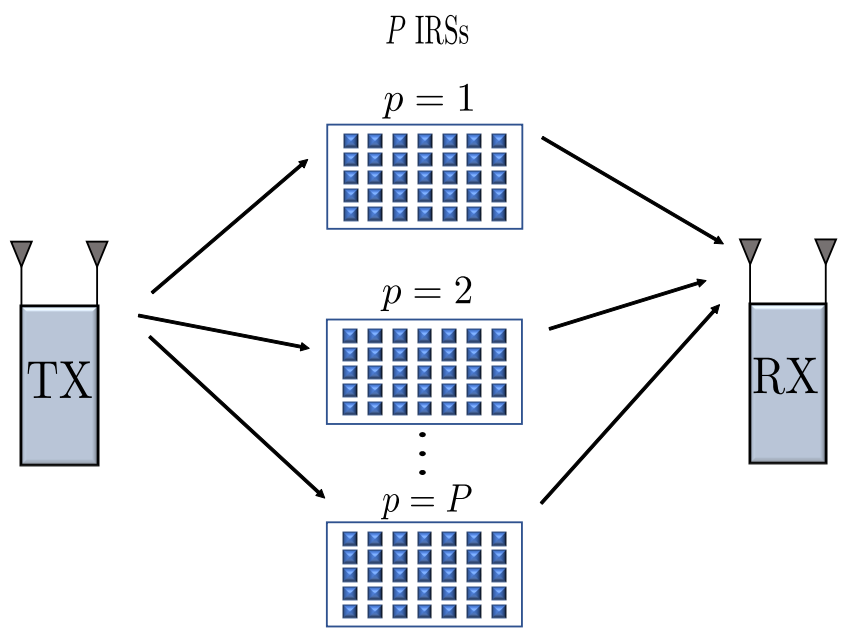

Fig. 1. MIMO communication system assisted by multiple IRSs. The signal from the transmitter to the receiver propagates via $P$ dominant scattererers. An IRS panel is placed at the exact location of each scatterer.

a vector into a diagonal matrix, $\operatorname{diag}_{j}(\mathbf{B})$ forms a diagonal matrix of size $R \times R$ out of the $j$ th column of $\mathbf{B}$. The $M \times M$ identity matrices is denoted by $\mathbf{I}_{M}$. The Kronecker product between two matrices is denoted by $\mathbf{Y}=\mathbf{A} \otimes \mathbf{B} \in$ $\mathbb{C}^{R I \times J J}$. The column-wise Kronecker product, also known as the Kathri-Rao product, is denoted by $\mathbf{Y}=\mathbf{A} \diamond \mathbf{B} \in \mathbb{C}^{R I \times J}$. In this paper, the following identities are used:

$$
\begin{aligned}
\operatorname{vec}(\mathbf{A B C}) & =\left(\mathbf{C}^{\mathrm{T}} \otimes \mathbf{A}\right) \operatorname{vec}(\mathbf{B}) \\
\operatorname{vec}(\mathbf{A} \mathbf{\Lambda} \mathbf{C}) & =\left(\mathbf{C}^{\mathrm{T}} \diamond \mathbf{A}\right) \boldsymbol{\lambda} \\
(\mathbf{A C}) \diamond(\mathbf{B D}) & =(\mathbf{A} \otimes \mathbf{B})(\mathbf{C} \diamond \mathbf{D})
\end{aligned}
$$

where all matrices have compatible dimensions and $\boldsymbol{\Lambda}=$ $\operatorname{diag}(\boldsymbol{\lambda})$.

\section{System Model}

Let us consider a MIMO system where the transmitter (TX) and receiver (RX) are equipped with uniform linear arrays (ULAs), having $M_{t}$ and $M_{r}$ antennas, respectively. We assume a specular multipath propagation environment with $P$ dominant scatterers. The received signal follows the wellknown input-output relation:

$$
\mathbf{y}=\mathbf{H x}+\mathbf{v} \in \mathbb{C}^{M_{r} \times 1}
$$

where $\mathbf{x} \in \mathbb{C}^{M_{t} \times 1}$ is the transmitted signal, $\mathbf{v} \in \mathbb{C}^{M_{r} \times 1}$ is the additive white Gaussian noise (AWGN) at the RX, while the channel matrix $\mathbf{H} \in \mathbb{C}^{M_{r} \times M_{t}}$ can be modeled as:

$$
\mathbf{H}=\sum_{p=1}^{P} \gamma_{p} \mathbf{a}_{p}^{(\mathrm{rx})} \mathbf{b}_{p}^{(\mathrm{tx}) T} \in \mathbb{C}^{M_{r} \times M_{t}},
$$

where $\gamma_{p}$ contains the path loss and the fast fading components associated with the $p$ th scatterer, $\mathbf{a}_{p}^{(\mathrm{rx})}$ and $\mathbf{b}_{p}^{(\mathrm{tx}) \mathrm{T}}$ are the $p$ th steering vectors, which are defined respectively as

$$
\begin{aligned}
& \mathbf{a}_{p}^{(\mathrm{rx})}=\left[1, \ldots, e^{-j \pi\left(M_{r}-1\right) \cos \left(\alpha_{p}\right)}\right]^{\mathrm{T}} \in \mathbb{C}^{M_{r} \times 1} \\
& \mathbf{b}_{p}^{(\mathrm{tx})}=\left[1, \ldots, e^{-j \pi\left(M_{t}-1\right) \cos \left(\beta_{p}\right)}\right]^{\mathrm{T}} \in \mathbb{C}^{M_{t} \times 1},
\end{aligned}
$$

where $\alpha_{p}$ and $\beta_{p}$ denote the angle of arrival (AOA) and angle of departure (AOD) of the $p$-th path, respectively.
Let us assume that these $P$ scatterers were identified, e.g., via some channel sounding method, and that it is possible to place $P$ IRSs at the location of these scatterers, as illustrated in Figure 1. Different from the usual IRS-aided MIMO system model considered in the literature [6]-[9], where the IRS is placed between the scatterers linking the TX and the RX, in this work we propose to study the effect of having multiple IRSs, each of which placed at the the exact location of the channel scatterers. Considering that the training sequences have a duration of $K \cdot P$ symbol periods as illustrated in Figure 2, the received pilot signals collected in the vector $\mathbf{y}_{. k} \in \mathbb{C}^{M_{r} \times 1}$ for the $k$ th time slot can be written as

$$
\mathbf{y}_{k}=\sum_{p=1}^{P} \gamma_{p} \mathbf{a}_{p}^{(\mathrm{rx})} \mathbf{a}_{p}^{(\mathrm{irs}) \mathrm{T}} \operatorname{diag}_{k}\left(\mathbf{S}^{(p)}\right) \mathbf{b}_{p}^{(\mathrm{irs})} \mathbf{b}_{p}^{(\mathrm{tx}) \mathrm{T}} \mathbf{x}_{k}+\mathbf{z}_{k}
$$

where $\mathbf{x}_{k} \in \mathbb{C}^{M_{r} \times 1}$ is the pilot symbol transmitted at the $k$ th time slot, $\mathbf{a}_{p}^{\text {(irs) }} \in \mathbb{C}^{N \times 1}$ and $\mathbf{b}_{p}^{\text {(irs) }} \in \mathbb{C}^{N \times 1}$ are the IRS array steering vectors associated with the $p$-th arrival and departure angles, respectively. Note that, since the IRS is a 2D panel, each AOA and AOD has azimuth and elevation responses. Based on the formulation of a uniform rectangular array (URA), we can write the arrival steering vector of the IRS as

$$
\mathbf{b}_{p}^{(\text {irs })}=\mathbf{b}_{p}^{v(\text { irs })} \otimes \mathbf{b}_{p}^{h(\text { irs })} \in \mathbb{C}^{N_{h} N_{v} \times 1}
$$

with $N_{h} N_{v}=N$, and $\mathbf{b}_{p}^{h \text { (irs) }}$ and $\mathbf{b}_{p}^{v \text { (irs) }}$ being the azimuth and elevation component vectors, respectively. The $n$th element of $\mathbf{b}_{p}^{\text {(irs) }}$ is given by

$$
\left[\mathbf{b}_{p}^{(\mathrm{irs})}\right]_{n_{h}+\left(n_{v}-1\right) N_{h}}=e^{j \pi\left(\left(n_{h}-1\right) \cos \phi_{p}^{b} \sin \psi_{p}^{b}+\left(n_{v}-1\right) \cos \phi_{p}^{b}\right)},
$$

with $n_{h}=\left\{1, \ldots, N_{h}\right\}, n_{v}=\left\{1, \ldots, N_{v}\right\}$, and $n=n_{h}+$ $\left(n_{v}-1\right) N_{h}$, where $\phi_{p}^{b}$ and $\psi_{p}^{b}$ are azimuth and elevation AOAs, respectively. Similarly, the departure steering vector of the IRS also has the same Kronecker structure, i.e.,

$$
\mathbf{a}_{p}^{(\text {irs })}=\mathbf{a}_{p}^{v(\text { irs })} \otimes \mathbf{a}_{p}^{h(\text { irs })} \in \mathbb{C}^{N_{h} N_{v} \times 1},
$$

where the $n$th element of $\mathbf{a}_{p}^{\text {(irs) }}$ is given by

$$
\left[\mathbf{a}_{p}^{(\mathrm{irs})}\right]_{n_{h}+\left(n_{v}-1\right) N_{h}}=e^{j \pi\left(\left(n_{h}-1\right) \cos \phi_{p}^{a} \sin \psi_{p}^{a}+\left(n_{v}-1\right) \cos \phi_{p}^{a}\right)},
$$

with $\phi_{p}^{a}$ and $\psi_{p}^{a}$ being the azimuth and elevation AODs, respectively.

In Equation (8), $\mathbf{S}^{(p)} \in \mathbb{C}^{N \times K}$ is the matrix that contains the IRS phase-shifts associated with the $p$-th IRS, which, for a certain time slot $k$, are collected in the following vector

$$
\mathbf{s}_{k}^{(p)}=\left[\beta_{1, k}^{(p)} e^{j \theta_{1, k}^{(p)}}, \ldots, \beta_{N, k}^{(p)} e^{j \theta_{N, k}^{(p)}}\right]^{\mathrm{T}} \in \mathbb{C}^{N \times 1}
$$

where $\beta_{n, k}=\{0,1\}$ and $\theta_{n, k}$ are the reflection coefficient and the designed phase-shift for the $n$th IRS element, respectively for the $k$ th time slot. 
Training Sequence: $P$ blocks of $K$ time slots

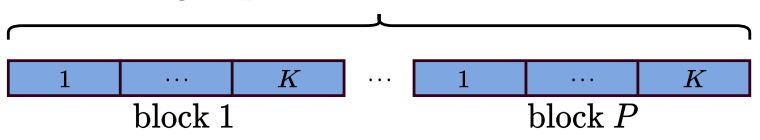

Fig. 2. Time-frame of channel estimation and IRS optimization.

\section{A. Channel Estimation and Joint Beamforming Design}

For the channel estimation and joint beamforming design, we propose the training sequence protocol shown in Figure 2, which is composed with $P$ blocks of size $K$ symbol periods. The main idea is to use these $P$ blocks to separate the contribution from the scatterers. First, let us rewrite Equation (8) as

$$
\begin{aligned}
\mathbf{y}_{k}^{(1)} & =\gamma_{1} \mathbf{a}_{1}^{(\mathrm{rx})} \mathbf{a}_{1}^{(\mathrm{irs}) \mathrm{T}} \operatorname{diag}_{k}\left(\mathbf{S}^{(1)}\right) \mathbf{b}_{1}^{(\mathrm{irs})} \mathbf{b}_{1}^{(\mathrm{tx}) \mathrm{T}} \mathbf{x}_{k}+\mathbf{v}_{k}^{(1)} \\
& +\underbrace{\sum_{p=2}^{P} \gamma_{p} \mathbf{a}_{p}^{(\mathrm{rx})} \mathbf{a}_{p}^{(\mathrm{irs}) \mathrm{T}} \operatorname{diag}_{k}\left(\mathbf{S}^{(p)}\right) \mathbf{b}_{p}^{(\mathrm{irs})} \mathbf{b}_{p}^{(\mathrm{tx}) \mathrm{T}} \mathbf{x}_{k}}_{\text {interference }} \in \mathbb{C}^{M_{r} \times 1}
\end{aligned}
$$

Let us assume that, in the first block, the desired signal is the one coming from the first IRS. Thus, the contribution from the remaining IRSs $(p=\{2, \ldots, P\})$ are considered as interference. To eliminate this interference, the reflecting coefficients $\beta_{n, k}^{(p)}$ from the IRSs placed at the scatterers $p=\{2, \ldots, P\}$ are set to zero. This means that they are in absorption mode ${ }^{1}$. Thus, for the first block $(p=1)$, the received pilots can be written as

$$
\mathbf{y}_{k}^{(1)}=\gamma_{1} \mathbf{a}_{1}^{(\mathrm{rx})} \mathbf{a}_{1}^{(\mathrm{irs}) \mathrm{T}} \operatorname{diag}_{k}\left(\mathbf{S}^{(1)}\right) \mathbf{b}_{1}^{(\mathrm{irs})} \mathbf{b}_{1}^{(\mathrm{tx}) \mathrm{T}} \mathbf{x}_{k}+\mathbf{v}_{k}^{(1)} .
$$

For the training stage, let us assume that all IRSs have the same phase-shift matrix, i.e., $\mathbf{S}^{(1)}=\ldots=\mathbf{S}^{(P)}=\mathbf{S} \in \mathbb{C}^{N \times K}$, and $\mathbf{S}$ is designed as a truncated DFT matrix. Neglecting the noise term, and applying properties (1), (2) and (3) to Eq. (10), we have

$$
\begin{aligned}
\mathbf{y}_{k}^{(1)} & =\gamma_{1}\left(\mathbf{x}_{k}^{\mathrm{T}} \otimes \mathbf{I}_{M_{r}}\right) \operatorname{vec}\left(\mathbf{a}_{1}^{(\mathrm{rx})} \mathbf{a}_{1}^{(\mathrm{irs}) \mathrm{T}} \operatorname{diag}_{k}\left(\mathbf{S}^{(1)}\right) \mathbf{b}_{1}^{(\mathrm{irs})} \mathbf{b}_{1}^{(\mathrm{tx}) \mathrm{T}}\right) \\
& =\gamma_{1}\left(\mathbf{x}_{k}^{\mathrm{T}} \otimes \mathbf{I}_{M_{r}}\right)\left(\left(\mathbf{b}_{1}^{(\mathrm{irs})} \mathbf{b}_{1}^{(\mathrm{tx}) \mathrm{T}}\right)^{\mathrm{T}} \diamond\left(\mathbf{a}_{1}^{(\mathrm{rx})} \mathbf{a}_{1}^{(\mathrm{irs}) \mathrm{T}}\right)\right) \mathbf{s}_{k}^{(1)} \\
& =\gamma_{1}\left(\mathbf{s}_{k}^{(1) \mathrm{T}} \otimes \mathbf{x}_{k}^{\mathrm{T}} \otimes \mathbf{I}_{M_{r}}\right) \operatorname{vec}\left(\left(\mathbf{b}_{1}^{(\mathrm{irs})} \mathbf{b}_{1}^{(\mathrm{tx}) \mathrm{T}}\right)^{\mathrm{T}} \diamond\left(\mathbf{a}_{1}^{(\mathrm{rx})} \mathbf{a}_{1}^{(\mathrm{irs}) \mathrm{T}}\right)\right) \\
& =\gamma_{1}\left(\mathbf{s}_{k}^{(1) \mathrm{T}} \otimes \mathbf{x}_{k}^{\mathrm{T}} \otimes \mathbf{I}_{M_{r}}\right) \operatorname{vec}\left(\left(\mathbf{b}_{1}^{(\mathrm{tx})} \otimes \mathbf{a}_{1}^{(\mathrm{rx})}\right)\left(\mathbf{b}_{1}^{(\mathrm{irs}) \mathrm{T}} \diamond \mathbf{a}_{1}^{(\mathrm{irs}) \mathrm{T}}\right)\right) \\
& =\left(\mathbf{s}_{k}^{(1) \mathrm{T}} \otimes \mathbf{x}_{k}^{\mathrm{T}} \otimes \mathbf{I}_{M_{r}}\right) \mathbf{z}^{(1)} .
\end{aligned}
$$

Stacking the $K$ vectors $\mathbf{y}_{1}^{(1)}, \ldots, \mathbf{y}_{K}^{(1)}$ associated with the first block as $\overline{\mathbf{y}}^{(1)}=\operatorname{vec}\left(\left[\mathbf{y}_{1}^{(1)}, \ldots, \mathbf{y}_{K}^{(1)}\right]\right) \in \mathbb{C}^{M_{R} K \times 1}$, and

\footnotetext{
${ }^{1}$ In the literature, most of the IRSs are built with resonant circuits in order to reflect the impinging electromagnetic waves to a specified direction [15]. However, in these circuits, the reflecting coefficient $\beta$ usually varies within 0.2 ( 0 degree phase-shift) up to 1 ( $\pi$ or $-\pi$ phase-shift) [15]. Nevertheless, the work of [16], shows that metamaterials are capable of perfectly absorbing the electromagnetic waves and reflect them to a specific direction at $\mathrm{THz}$ frequencies.
}

adding the noise term back, we have

$$
\overline{\mathbf{y}}^{(1)}=\mathbf{C z}^{(1)}+\mathbf{v}^{(1)} \in \mathbb{C}^{M_{r} K \times 1},
$$

where $\left.\mathbf{v}^{(1)}=\left[\mathbf{v}_{1}^{(1)}, \ldots, \mathbf{v}_{K}^{(1)}\right], \mathbf{C}=\left[(\mathbf{S} \diamond \mathbf{X}) \otimes \mathbf{I}_{M_{r}}\right)\right]^{\mathrm{T}} \in$ $\mathbb{C}^{M_{r} K \times M_{r} M_{t} N}$ is the matrix containing the known system parameters, such as the pre-defined phase-shift matrix $\mathbf{S}$ (truncated DFT matrix) and $\mathbf{X} \in \mathbb{C}^{M_{t} \times K}$ (the pilot signal matrix). On the other hand, $\mathbf{z}^{(1)}=\gamma_{1} \operatorname{vec}\left(\left(\mathbf{b}^{(\mathrm{tx})} \otimes \mathbf{a}^{(\mathrm{rx})}\right)\left(\mathbf{b}_{1}^{(\mathrm{irs}) \mathrm{T}} \diamond \mathbf{a}_{1}^{(\mathrm{irs}) \mathrm{T}}\right)\right)$ is the parameter vector to be estimated, which combines the channel gains and the array steering vectors from the TX, RX and the IRS. A least square (LS) estimate of $\mathbf{z}^{(1)}$ can be obtained from Equation (12) as

$$
\hat{\mathbf{z}}^{(1)} \approx \mathbf{C}^{+} \overline{\mathbf{y}}_{1} \in \mathbb{C}^{M_{r} M_{t} N \times 1} .
$$

It is important to note that, to ensure $\mathbf{C}$ is left-invertible, we need to satisfy the condition $K \geq N M_{t}$.

Defining $\hat{\mathbf{Z}}^{(1)}=$ unvec $_{M_{r} M_{t} \times N}\left(\hat{\mathbf{z}}^{(1)}\right)$, we have

$$
\begin{aligned}
\hat{\mathbf{Z}}^{(1)} & \approx \gamma_{1}\left(\mathbf{b}_{1}^{(\mathrm{tx})} \otimes \mathbf{a}_{1}^{(\mathrm{rx})}\right)\left(\mathbf{b}_{1}^{(\mathrm{irs}) \mathrm{T}} \diamond \mathbf{a}_{1}^{(\mathrm{irs}) \mathrm{T}}\right) \in \mathbb{C}^{M_{r} M_{t} \times N} \\
& =\mathbf{f}_{1} \mathbf{r}_{1}^{\mathrm{T}} .
\end{aligned}
$$

Note that $\hat{\mathbf{Z}}^{(1)}$ is approximately a rank-one matrix, thus, defining $\hat{\mathbf{Z}}^{(1)}=\mathbf{U}^{(1)} \boldsymbol{\Sigma}^{(1)} \mathbf{V}^{(1) \mathrm{H}}$ as the singular value decomposition (SVD) of $\hat{\mathbf{Z}}^{(1)}$, we can obtain estimates of $\mathbf{f}_{1}$ and $\mathbf{r}_{1}$ from its dominant left and right singular vectors, respectively

$$
\begin{aligned}
\hat{\mathbf{f}}_{1} & =\mathbf{u}_{1}^{(1)} \in \mathbb{C}^{M_{r} M_{t} \times 1} \\
\hat{\mathbf{r}}_{1} & =\mathbf{v}_{1}^{(1) *} \in \mathbb{C}^{N \times 1}
\end{aligned}
$$

The optimum phase-shifts of the first IRS are obtained as

$$
\mathbf{s}_{\text {opt }}^{(1)}=e^{-j \angle \hat{\mathbf{r}}_{1}} \in \mathbb{C}^{N \times N} .
$$

From $\hat{\mathbf{f}}_{1}$, we can obtain an estimate of the first column of the steering matrices $\mathbf{A}^{(\mathrm{rx})}$ and $\mathbf{B}^{(\mathrm{tx})}$, and since $\hat{\mathbf{f}}_{1} \approx \mathbf{b}_{1}^{(\mathrm{tx})} \otimes \mathbf{a}_{1}^{(\mathrm{rx})} \in$ $\mathbb{C}^{M_{r} M_{t}}$. To this end, defining $\hat{\mathbf{F}}_{1}=\operatorname{unvec}_{M r \times M_{t}}\left(\hat{\mathbf{f}}_{1}\right)$ yields

$$
\hat{\mathbf{F}}_{1} \approx \mathbf{a}_{1}^{(\mathrm{rx})} \cdot \mathbf{b}_{1}^{(\mathrm{tx}) \mathrm{T}}
$$

Computing the SVD of $\hat{\mathbf{F}}_{1}=\mathbf{U}^{\mathrm{f}(1)} \boldsymbol{\Sigma}^{\mathrm{f}(1)} \mathbf{V}^{\mathrm{f}(1) \mathrm{H}}$, the estimates of $\mathbf{a}_{1}^{(\mathrm{rx})}$ and $\mathbf{b}^{(\mathrm{tx})}$ are obtained, respectively, as

$$
\begin{aligned}
& \hat{\mathbf{a}}_{1}^{(\mathrm{rx})}=\mathbf{u}_{1}^{\mathrm{f}(1)} \in \mathbb{C}^{M_{r} \times 1} \\
& \hat{\mathbf{b}}_{1}^{(\mathrm{tx})}=\mathbf{v}_{1}^{\mathrm{f}(1) *} \in \mathbb{C}^{M_{t} \times 1}
\end{aligned}
$$

After repeating the procedure from Equations (13) to (18) for all $P$ blocks, the estimated transmit and receive steering matrices the TX and RX, respectively, are built as

$$
\begin{aligned}
& \hat{\mathbf{A}}^{(\mathrm{rx})}=\left[\hat{\mathbf{a}}_{1}^{(\mathrm{rx})}, \ldots, \hat{\mathbf{a}}_{P}^{(\mathrm{rx})}\right] \in \mathbb{C}^{M_{r} \times P} \\
& \hat{\mathbf{B}}^{(\mathrm{tx})}=\left[\hat{\mathbf{b}}_{1}^{(\mathrm{tx})}, \ldots, \hat{\mathbf{b}}_{P}^{(\mathrm{tx})}\right] \in \mathbb{C}^{M_{t} \times P} .
\end{aligned}
$$

Therefore, the total training overhead is given by $K \cdot P$ symbol periods, or, considering its minimum value, we have $N \cdot M_{t} \cdot P$ symbol periods. The proposed channel estimation and beamforming design procedure are summarized in Algorithm 1. 


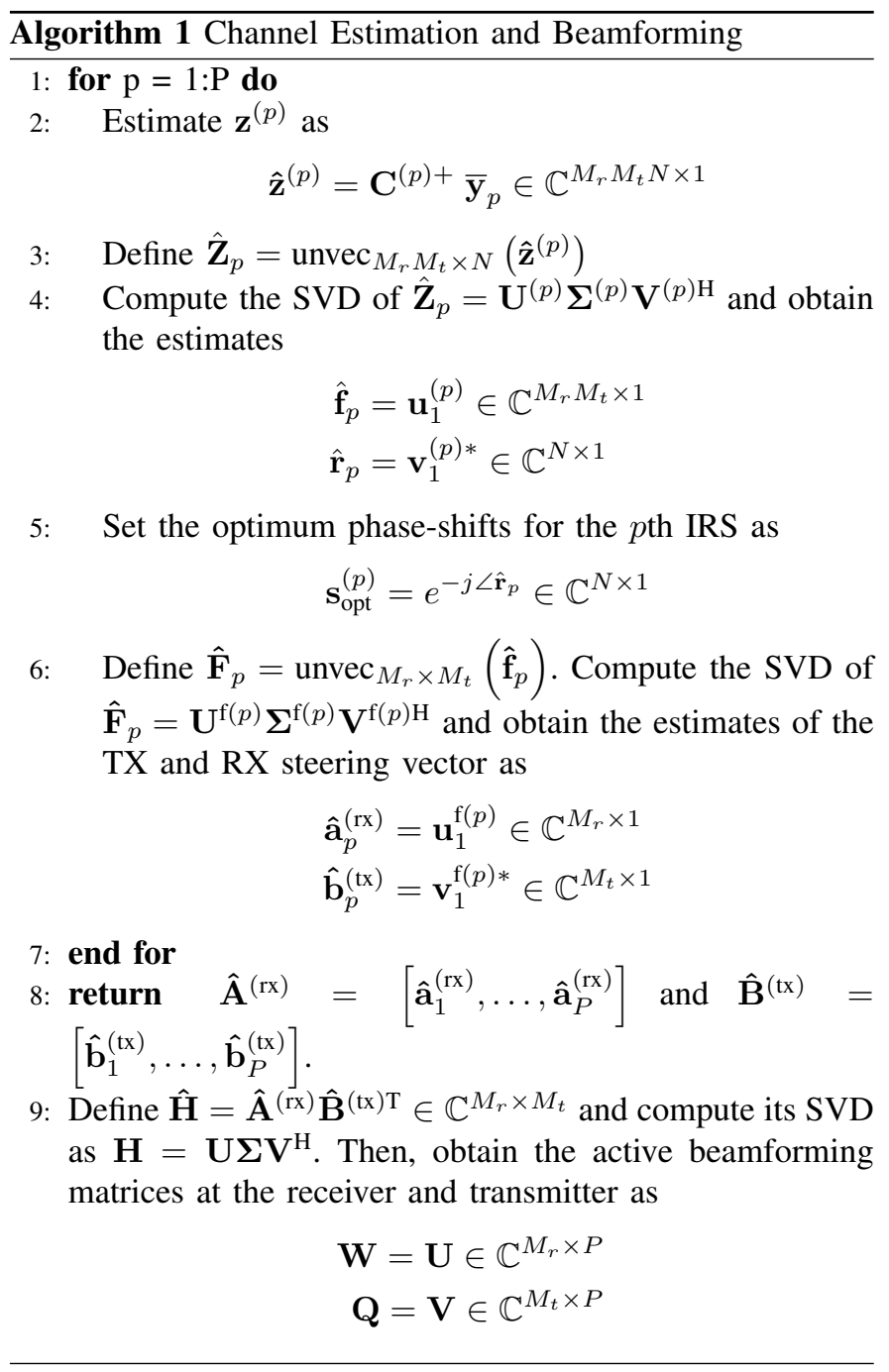

\section{B. Data transmission}

After the optimization of the IRS and the active beamforming matrices, data transmission starts. The received signal is then given by $\mathbf{y}^{(\mathrm{d})}=\mathbf{W}^{\mathrm{H}} \mathbf{A}^{\mathrm{rx}} \operatorname{diag}(\boldsymbol{\lambda}) \operatorname{diag}(\boldsymbol{\mu}) \mathbf{B}^{(\mathrm{tx}) \mathrm{T}} \mathbf{Q} \mathbf{x}^{(\mathrm{d})}+\mathbf{W}^{\mathrm{H}} \mathbf{v}^{(\mathrm{d})} \in \mathbb{C}^{P \times 1}$,

where $\mathbf{v}^{(\mathrm{d})} \sim \mathcal{C N}\left(0, \sigma_{\mathrm{d}}^{2}\right)$ is the AWGN at the receiver, $\gamma=\left[\gamma_{1}, \ldots, \gamma_{P}\right]^{\mathrm{T}} \in \mathbb{C}^{P \times 1}$ is the vector containing the path loss and fast fading between the TX and RX, while $\boldsymbol{\mu}=\left[\mu_{1}, \ldots, \mu_{P}\right]^{\mathrm{T}} \in \mathbb{C}^{P \times 1}$ is the path gain provided by the IRSs, which can be written as

$$
\boldsymbol{\mu}=\left[\begin{array}{c}
\mathbf{a}_{1}^{(\mathrm{irs}) \mathrm{T}} \operatorname{diag}\left(\mathbf{s}_{\mathrm{opt}}^{(1)}\right) \mathbf{b}_{1}^{(\mathrm{irs})} \\
\vdots \\
\mathbf{a}_{P}^{(\mathrm{irs}) \mathrm{T}} \operatorname{diag}\left(\mathbf{s}_{\mathrm{opt}}^{(P)}\right) \mathbf{b}_{P}^{(\mathrm{irs})}
\end{array}\right] \in \mathbb{C}^{P \times 1}
$$

\section{Simulation RESUlTS}

In this section, we evaluate the performance of the proposed scheme in terms of spectral efficiency by comparing the proposed scheme with the one without the IRS. The transmitted

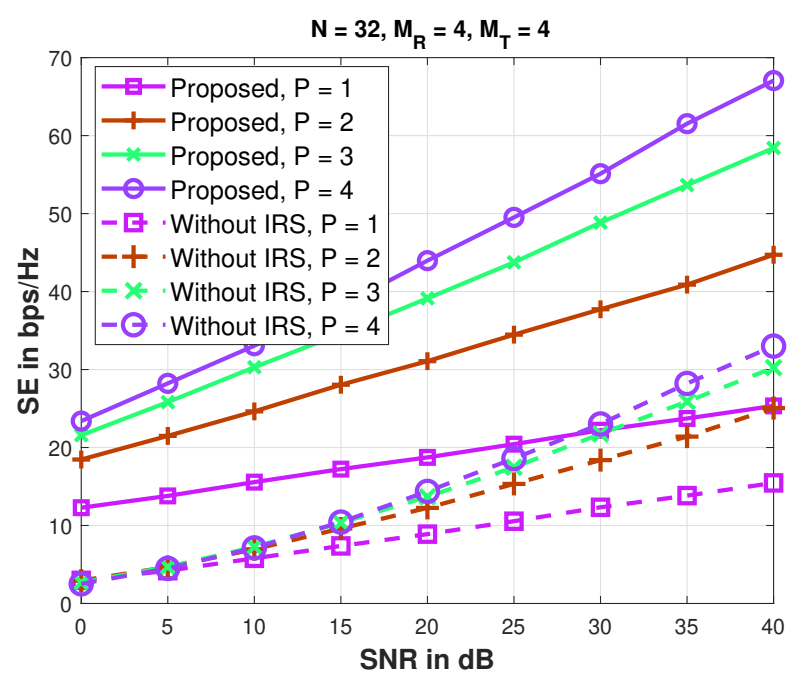

Fig. 3. Spectral efficiency for different values of $P$.

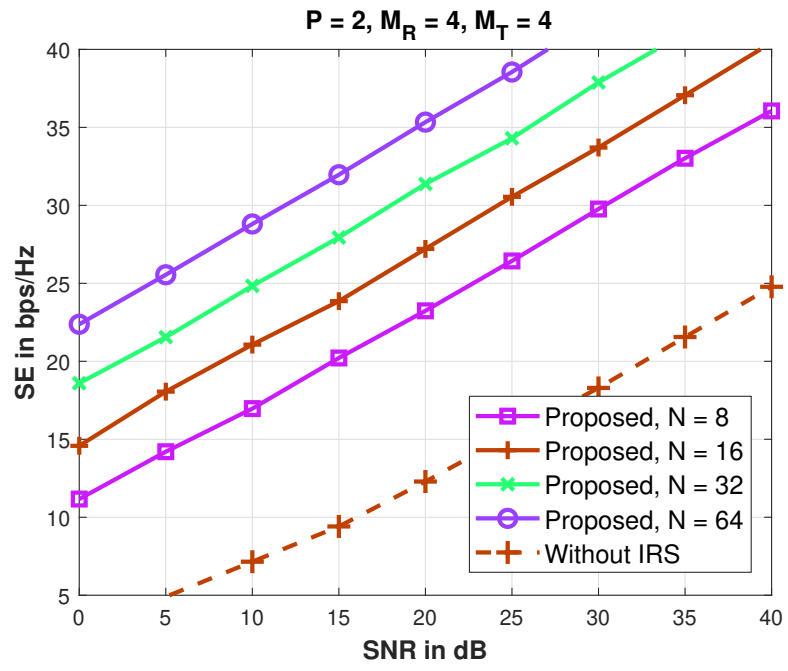

Fig. 4. Spectral efficiency for $N$ IRS elements.

symbols are normalized such that $\mathbb{E}\left\{\mathbf{x}^{(\mathrm{d})} \mathbf{x}^{(\mathrm{d}) H}\right\}=1$. The SNR is controlled by varying the noise power $\sigma_{\mathrm{d}}^{2}$, i.e., SNR $=1 / \sigma_{\mathrm{d}}^{2}$. The AOA $\alpha_{p}$ and AOD $\beta_{p}$ are randomly generated from an uniform distribution with values between $[-\pi, \pi]$. At the IRS, the elevation angles of departure and arrival are generated from an uniform distribution between $[-\pi / 2, \pi / 2]$. The combined effect of path loss and fast fading components is modeled as Gaussian random variables with $\gamma_{p} \sim \mathcal{C N}(0,1)$. The equivalent MIMO channel (including the transmit and receive beamformings) is given as

$$
\mathbf{H}_{\mathrm{eq}}=\mathbf{W}^{\mathrm{H}} \mathbf{A}^{\mathrm{rx}} \operatorname{diag}(\boldsymbol{\lambda}) \operatorname{diag}(\boldsymbol{\mu}) \mathbf{B}^{(\mathrm{tx}) \mathrm{T}} \mathbf{Q}
$$

The spectral efficiency is then calculated as

$$
\mathrm{SE}(\mathrm{bps} / \mathrm{Hz})=\log 2\left[\operatorname{det}\left(\mathbf{I}_{P}+\frac{\mathbf{H}_{\mathrm{eq}} \mathbf{R}_{x x} \mathbf{H}_{\mathrm{eq}}^{\mathrm{H}}}{\sigma_{\mathrm{d}}^{2}}\right)\right],
$$

with $\mathbf{R}_{x x}=\mathbb{E}\left\{\mathbf{x}^{(\mathrm{d})} \mathbf{x}^{(\mathrm{d}) H}\right\}$, and trace $\left(\mathbf{Q} \mathbf{R}_{x x} \mathbf{Q}^{\mathrm{H}}\right)=1$.

In Figure 3, we can observe the impact of deploying multiple IRSs in the considered MIMO communication system. 


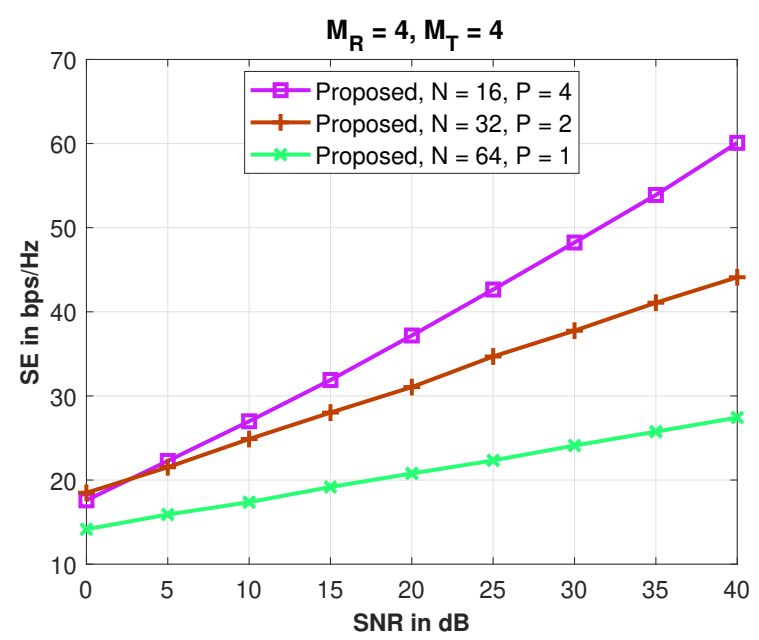

Fig. 5. Spectral efficiency for different values of $N$ and $P$ in order to ensure the same training overhead for all cases.

When $P=4$ and SNR is equal to $20 \mathrm{~dB}$, the proposed multiIRS scheme achieves an SE of $44 \mathrm{bps} / \mathrm{Hz}$ approximately, while for the one without IRS, the SE is approximately $14 \mathrm{bps} / \mathrm{Hz}$. This is clear, since as $P$ increases we have more IRSs in the system to perform a smart passive beamforming. However, it is important to note that, as $P$ increases, the training overhead also increases since $K \cdot P$ is required. At high SNR values ( $>30 \mathrm{~dB}$ ), the multi-IRS scheme is outperformed by the single-IRS one $(P=1)$.

In Figure 4, we compare the proposed scheme by fixing $P=2$, while varying the number $N$ of reflecting elements per IRS. As expected, the SE increases with the increase of $N$. As a reference for comparison, we also plot the spectral efficiency for the case without IRS. We notice that, even for a small number of reflecting elements $(N=8)$, the proposed scheme outperforms the one without IRS. The result of Figure 5 shows a spectral efficiency comparison of the proposed scheme with three different configurations that have the same training overhead, i.e., $K \cdot P$, that is equivalent to $N \cdot M_{t} \cdot P$. We can observe that, the setup with $N=16$ and $P=4$ has a better performance than the one with $N=64$ and $P=1$. This is an interesting result. One may conclude from this figure, is that in the proposed scenario, it is preferable to have $P$ IRSs with $N$ reflecting elements each, than a single IRS with $P \cdot N$ elements, in order to enjoy the diversity present in the system.

\section{Conclusion And Perspectives}

In this paper, we have proposed a channel estimation and joint beamforming design for a MIMO multi-IRS system, where multiple IRSs are placed on the exact location of the system scatterers. By separating the scatterers with the help of the IRSs, the channel is estimated and the active and passive beamformings are designed. Since each IRS is assumed to be individually aligned with a different scatterer, an optimum multipath diversity gain is efficiently exploited. In later works we shall investigate the case when the number of scatterers is larger than the possible number of IRSs deployment in the system. Also, we aim at considering a scenario with interference among the IRSs.

\section{REFERENCES}

[1] S. Gong, X. Lu, D. T. Hoang, D. Niyato, L. Shu, D. I. Kim, and Y.-C. Liang, "Toward smart wireless communications via intelligent reflecting surfaces: A contemporary survey," IEEE Communications Surveys \& Tutorials, vol. 22, no. 4, pp. 2283-2314, 2020.

[2] C. Liaskos, S. Nie, A. Tsioliaridou, A. Pitsillides, S. Ioannidis, and I. Akyildiz, "A new wireless communication paradigm through softwarecontrolled metasurfaces," IEEE Communications Magazine, vol. 56 , no. 9, pp. 162-169, 2018

[3] S. S. Bukhari, J. Y. Vardaxoglou, and W. Whittow, "A metasurfaces review: Definitions and applications," Applied Sciences, vol. 9, no. 13, p. $2727,2019$.

[4] Q. Wu, S. Zhang, B. Zheng, C. You, and R. Zhang, "Intelligent reflecting surface aided wireless communications: A tutorial," IEEE Transactions on Communications, 2021.

[5] E. Björnson, Ö. Özdogan, and E. G. Larsson, "Intelligent reflecting surface versus decode-and-forward: How large surfaces are needed to beat relaying?," IEEE Wireless Communications Letters, vol. 9, no. 2, pp. 244-248, 2019.

[6] G. T. de Araújo, A. L. De Almeida, and R. Boyer, "Channel estimation for intelligent reflecting surface assisted mimo systems: A tensor modeling approach," IEEE Journal of Selected Topics in Signal Processing, vol. 15 , no. 3, pp. 789-802, 2021.

[7] L. Wei, C. Huang, G. C. Alexandropoulos, C. Yuen, Z. Zhang, and M. Debbah, "Channel estimation for ris-empowered multi-user MISO wireless communications," IEEE Transactions on Communications, 2021.

[8] K. Ardah, S. Gherekhloo, A. L. de Almeida, and M. Haardt, "Trice: A channel estimation framework for ris-aided millimeter-wave MIMO systems," IEEE Signal Processing Letters, vol. 28, pp. 513-517, 2021.

[9] Z. Wang, L. Liu, and S. Cui, "Channel estimation for intelligent reflecting surface assisted multiuser communications," in 2020 IEEE Wireless Communications and Networking Conference (WCNC), pp. 16, IEEE, 2020

[10] Ö. Özdogan, E. Björnson, and E. G. Larsson, "Intelligent reflecting surfaces: Physics, propagation, and pathloss modeling," IEEE Wireless Communications Letters, vol. 9, no. 5, pp. 581-585, 2019.

[11] E. Björnson, Ö. Özdogan, and E. G. Larsson, "Reconfigurable intelligent surfaces: Three myths and two critical questions," IEEE Communications Magazine, vol. 58, no. 12, pp. 90-96, 2020.

[12] P. Wang, J. Fang, X. Yuan, Z. Chen, and H. Li, "Intelligent reflecting surface-assisted millimeter wave communications: Joint active and passive precoding design," IEEE Transactions on Vehicular Technology, 2020.

[13] T. L. Jensen and E. De Carvalho, "An optimal channel estimation scheme for intelligent reflecting surfaces based on a minimum variance unbiased estimator," in ICASSP 2020-2020 IEEE International Conference on Acoustics, Speech and Signal Processing (ICASSP), pp. 5000-5004, IEEE, 2020.

[14] A. Zappone, M. Di Renzo, F. Shams, X. Qian, and M. Debbah, "Overhead-aware design of reconfigurable intelligent surfaces in smart radio environments," IEEE Transactions on Wireless Communications, 2020.

[15] S. Abeywickrama, R. Zhang, Q. Wu, and C. Yuen, "Intelligent reflecting surface: Practical phase shift model and beamforming optimization," IEEE Transactions on Communications, vol. 68, no. 9, pp. 5849-5863, 2020.

[16] T. Badloe, J. Mun, and J. Rho, "Metasurfaces-based absorption and reflection control: perfect absorbers and reflectors," Journal of Nanomaterials, vol. 2017, 2017. 